\title{
Badly approximable numbers and Littlewood-type problems
}

\author{
Yann Bugeaud \& Nikolay Moshchevitin
}

\begin{abstract}
We establish that the set of pairs $(\alpha, \beta)$ of real numbers such that

$$
\liminf _{q \rightarrow+\infty} q \cdot(\log q)^{2} \cdot\|q \alpha\| \cdot\|q \beta\|>0,
$$

where $\|\cdot\|$ denotes the distance to the nearest integer, has full Hausdorff dimension in $\mathbf{R}^{2}$. Our proof rests on a method introduced by Peres and Schlag, that we further apply to various Littlewood-type problems.
\end{abstract}

\section{Introduction}

A famous open problem in simultaneous Diophantine approximation, called the Littlewood conjecture [14], claims that, for any given pair $(\alpha, \beta)$ of real numbers, we have

$$
\inf _{q \geq 1} q \cdot\|q \alpha\| \cdot\|q \beta\|=0,
$$

where $\|\cdot\|$ denotes the distance to the nearest integer. Throughout the present paper, we denote by $\boldsymbol{B} \boldsymbol{a d}$ the set of badly approximable numbers, that is,

$$
\boldsymbol{B a d}=\left\{\alpha \in \mathbf{R}: \inf _{q \geq 1} q \cdot\|q \alpha\|>0\right\},
$$

and we recall that $\boldsymbol{B a d}$ has Lebesgue measure zero and full Hausdorff dimension [12]. Consequently, (1.1) holds for almost every pair $(\alpha, \beta)$ of real numbers. Recently, this result was considerably improved by Einsiedler, Katok and Lindenstrauss [8], who established that the set of pairs $(\alpha, \beta)$ for which (1.1) do not hold has Hausdorff dimension zero; see also [24] for a weaker statement, and Section 10.1 of [4] for a survey of related results.

Another metrical statement connected to the Littlewood conjecture was established by Gallagher [11] in 1962 and can be formulated as follows (see e.g. [2]).

Theorem G. Let $n$ be a positive integer. Let $\Psi: \mathbf{R}_{>0} \rightarrow \mathbf{R}_{>0}$ be a non-increasing function. The set of points $\left(x_{1}, \ldots, x_{n}\right)$ in $\mathbf{R}^{n}$ such that there are infinitely many positive integers $q$ satisfying

$$
\prod_{i=1}^{n}\left\|q x_{i}\right\|<\Psi(q)
$$

2000 Mathematics Subject Classification: 11J13; 11J25, 11 K60 . 
has full Lebesgue measure if the sum

$$
\sum_{h \geq 1} \Psi(h)^{n}(\log h)^{n-1}
$$

diverges, and has zero Lebesgue measure otherwise.

In particular, it follows from Gallagher's theorem that

$$
\liminf _{q \rightarrow+\infty} q \cdot(\log q)^{2} \cdot\|q \alpha\| \cdot\|q \beta\|=0
$$

for almost every pair $(\alpha, \beta)$ of real numbers. The main purposes of the present note are to establish the existence of exceptional pairs $(\alpha, \beta)$ which do not satisfy (1.2) - a result first proved in [21] - , and to prove that the set of these pairs has full Hausdorff dimension in $\mathbf{R}^{2}$. We further consider various questions closely related to the Littlewood conjecture.

Our main results are stated in Section 2 and proved in Sections 4 and 5, with the help of auxiliary lemmas gathered in Section 3. Several additional results are given in Section 6 .

Throughout this paper, $\lfloor x\rfloor$ and $\lceil x\rceil$ denote the greatest integer less than or equal to $x$ and the smallest integer greater than or equal to $x$, respectively.

\section{Main results}

Our first result shows that there are many pairs $(\alpha, \beta)$ of real numbers that are not well multiplicatively approximable.

Theorem 1. For every real number $\alpha$ in $\boldsymbol{B a d}$, the set of real numbers $\beta$ such that

$$
\liminf _{q \rightarrow+\infty} q \cdot(\log q)^{2} \cdot\|q \alpha\| \cdot\|q \beta\|>0
$$

has full Hausdorff dimension.

The proof of Theorem 1 uses a method introduced by Peres and Schlag [23], which was subsequently applied in $[17,18,19,20,21]$.

Since the set $\boldsymbol{B a d}$ has full Hausdorff dimension, the next result follows from Theorem 1 by an immediate application of Corollary 7.12 from [10].

Theorem 2. The set of pairs $(\alpha, \beta)$ of real numbers satisfying

$$
\liminf _{q \rightarrow+\infty} q \cdot(\log q)^{2} \cdot\|q \alpha\| \cdot\|q \beta\|>0
$$

has full Hausdorff dimension in $\mathbf{R}^{2}$.

Theorem 1 can be viewed as a complement to the following result of Pollington and Velani [24]. 
Theorem PV. For every real number $\alpha$ in $\boldsymbol{B a d}$, there exists a subset $G(\alpha)$ of $\boldsymbol{B a d}$ with full Hausdorff dimension such that, for any $\beta$ in $G(\alpha)$, there exist arbitrarily large integers $q$ satisfying

$$
q \cdot(\log q) \cdot\|q \alpha\| \cdot\|q \beta\| \leq 1 .
$$

In [1], the authors constructed explicitly for every $\alpha$ in $\boldsymbol{B a d}$ uncountably many $\beta$ in Bad such that the pair $(\alpha, \beta)$ satisfies (1.1), and even a strong form of this inequality. It would be very interesting to construct explicit examples of pairs of real numbers that satisfy $(2.1)$.

A modification of an auxiliary lemma yields a slight improvement on Theorem 1.

Theorem 3. Let $a$ be a real number with $0<a<1$. For every real number $\alpha$ in $\boldsymbol{B a d}$, the set of real numbers $\beta$ such that

$$
\liminf _{q \rightarrow+\infty} q \cdot(\log q)^{2-a} \cdot(\log 1 /\|q \alpha\|)^{a} \cdot\|q \alpha\| \cdot\|q \beta\|>0
$$

has full Hausdorff dimension.

Theorem 3 is stronger than Theorem 1 since, for every $\alpha$ in $\boldsymbol{B a d}$, there exists a positive real number $\delta$ such that $\log (1 /\|q \alpha\|) \leq \delta \log q$ holds for every integer $q \geq 2$.

Cassels and Swinnerton-Dyer [7] proved that (1.1) is equivalent to the equality

$$
\inf _{(x, y) \in \mathbf{Z} \times \mathbf{Z} \backslash\{(0,0)\}} \max \{|x|, 1\} \cdot \max \{|y|, 1\} \cdot\|x \alpha+y \beta\|=0,
$$

and used it to show that (1.1) holds if $\alpha$ and $\beta$ belong to the same cubic number field (see also [22]). In this context, we have the following metrical result, extracted from page 455 of [3]. For integers $q_{1}, \ldots, q_{n}$, set

$$
\Pi\left(q_{1}, \ldots, q_{n}\right)=\prod_{i=1}^{n} \max \left\{1,\left|q_{i}\right|\right\} .
$$

Theorem BKM. Let $n$ be a positive integer. Let $\Psi: \mathbf{R}_{>0} \rightarrow \mathbf{R}_{>0}$ be a non-increasing function. The set of points $\left(x_{1}, \ldots, x_{n}\right)$ in $\mathbf{R}^{n}$ such that there are infinitely many integers $q_{1}, \ldots, q_{n}$ satisfying

$$
\left\|q_{1} x_{1}+\ldots+q_{n} x_{n}\right\|<\Psi\left(\Pi\left(q_{1}, \ldots, q_{n}\right)\right)
$$

has full Lebesgue measure if the sum

$$
\sum_{h \geq 1} \Psi(h)(\log h)^{n-1}
$$

diverges, and has zero Lebesgue measure otherwise.

For $n \geq 2$, there is no known example of points $\left(x_{1}, \ldots, x_{n}\right)$ in $\mathbf{R}^{n}$ and of a function $\Psi$ as in Theorem BKM such that the sum (2.3) diverges and (2.2) has only finitely many solutions. The Peres-Schlag method allows us to show that such examples do exist. 
Theorem 4. The set of pairs $(\alpha, \beta)$ of real numbers satisfying

$$
\liminf _{x, y \geq 0} \max \{2,|x y|\} \cdot\|x \alpha+y \beta\| \cdot(\log \max \{2,|x y|\})^{2}>0
$$

has full Hausdorff dimension in $\mathbf{R}^{2}$.

The proof of Theorem 4 is briefly outlined in Section 5. Note that Theorem 4 (resp. Theorem 1) does not follow from Theorem 1 (resp. Theorem 4) by some transference principle.

In analogy with the Littlewood conjecture, de Mathan and Teulié [16] proposed recently a 'mixed Littlewood conjecture'. For any prime number $p$, the usual $p$-adic absolute value $|\cdot|_{p}$ is normalized in such a way that $|p|_{p}=p^{-1}$.

De Mathan-Teulié conjecture. For every real number $\alpha$ and every prime number $p$, we have

$$
\inf _{q \geq 1} q \cdot\|q \alpha\| \cdot|q|_{p}=0 .
$$

Despite several recent results $[9,5]$, this conjecture is still unsolved. The following metrical statement, established in [6], should be compared with Theorem G.

Theorem BHV. Let $k$ be a positive integer. Let $p_{1}, \ldots, p_{k}$ be distinct prime numbers. Let $\Psi: \mathbf{R}_{>0} \rightarrow \mathbf{R}_{>0}$ be a non-increasing function. The set of real numbers $\alpha$ such that there are infinitely many positive integers $q$ satisfying

$$
\|q \alpha\| \cdot|q|_{p_{1}} \cdots|q|_{p_{k}}<\Psi(q)
$$

has full Lebesgue measure if the sum

$$
\sum_{h \geq 1} \Psi(h)(\log h)^{k}
$$

diverges, and has zero Lebesgue measure otherwise.

As an immediate consequence of Theorem BHV, we get that, for every prime number $p$, almost every real number $\alpha$ satisfies

$$
\inf _{q \geq 2} q \cdot(\log q)^{2} \cdot(\log \log q) \cdot\|q \alpha\| \cdot|q|_{p}=0
$$

The method of proof of Theorem 1 allows us to confirm the existence of real numbers for which (2.4) does not hold.

Theorem 5. Let $a$ be a real number with $0 \leq a<1$. For every prime number $p$, the set of real numbers $\alpha$ such that

$$
\liminf _{q \rightarrow+\infty} q \cdot(\log q)^{2-a} \cdot\|q \alpha\| \cdot|q|_{p} \cdot\left(\log 2 /|q|_{p}\right)^{a}>0
$$

has full Hausdorff dimension.

We display an immediate consequence of Theorem 5. 
Corollary 1. For every prime number $p$, the set of real numbers $\alpha$ such that

$$
\liminf _{q \rightarrow+\infty} q \cdot(\log q)^{2} \cdot\|q \alpha\| \cdot|q|_{p}>0
$$

has full Hausdorff dimension.

In the present note, we have restricted our attention to 2-dimensional questions. However, our method can be successfully applied to prove that, given an integer $n \geq 2$, there are real numbers $\alpha_{1}, \ldots, \alpha_{n}$ such that

$$
\liminf _{q \rightarrow+\infty} q \cdot(\log q)^{n} \cdot\left\|q \alpha_{1}\right\| \cdots\left\|q \alpha_{n}\right\|>0
$$

as well as real numbers $\beta_{1}, \ldots, \beta_{n}$ such that

$$
\liminf _{x_{1}, \ldots, x_{n} \geq 0} \max \left\{2,\left|x_{1} \ldots x_{n}\right|\right\} \cdot\left\|x_{1} \beta_{1}+\ldots+x_{n} \beta_{n}\right\| \cdot\left(\log \max \left\{2,\left|x_{1} \ldots x_{n}\right|\right\}\right)^{n}>0
$$

This will be the subject of subsequent work by E. Ivanova.

\section{Auxiliary results}

The original method of Peres and Schlag is a construction of nested intervals. A useful tool for estimating from below the Hausdorff measure of a Cantor set is the mass distribution principle, which we recall now.

We consider a set $\mathcal{K}$ included in a bounded interval $E$, and defined as follows. Set $\mathcal{E}_{0}=E$ and assume that, for any positive integer $k$, there exists a finite family $\mathcal{E}_{k}$ of disjoint compact intervals in $E$ such that any interval $U$ belonging to $\mathcal{E}_{k}$ is contained in exactly one of the intervals of $\mathcal{E}_{k-1}$ and contains at least two intervals belonging to $\mathcal{E}_{k+1}$. Suppose also that the maximum of the lengths of the intervals in $\mathcal{E}_{k}$ tends to 0 when $k$ tends to infinity. For $k \geq 0$, denote by $E_{k}$ the union of the intervals belonging to the family $\mathcal{E}_{k}$, and set

$$
\mathcal{K}:=\bigcap_{k=1}^{+\infty} E_{k}
$$

Lemma 1. Keep the same notation as above. Assume further that there exists a positive integer $k_{0}$ such that, for any $k \geq k_{0}$, each interval of $E_{k-1}$ contains at least $m_{k} \geq 2$ intervals of $E_{k}$, these being separated by at least $\varepsilon_{k}$, where $0<\varepsilon_{k+1}<\varepsilon_{k}$. We then have

$$
\operatorname{dim} \mathcal{K} \geq \liminf _{k \rightarrow+\infty} \frac{\log \left(m_{1} \ldots m_{k-1}\right)}{-\log \left(m_{k} \varepsilon_{k}\right)}
$$

Proof. This is Example 4.6 in [10], see also Proposition 5.2 in [4].

Lemma 2. Let $\alpha$ be in Bad. There exists a positive constant $C(\alpha)$ such that, for every integer $q \geq 2$, we have

$$
\sum_{x=q}^{q^{3}} \frac{1}{\|\alpha x\| x \log _{2}^{2} x} \leq C(\alpha) .
$$


Proof. This is a straightforward consequence of Example 3.2 on page 124 of [13], where it is established that there exists a positive constant $C_{1}(\alpha)$ such that

$$
\sum_{x=1}^{m} \frac{1}{\|\alpha x\| x} \leq C_{1}(\alpha)(\log m)^{2}
$$

for all positive integers $m$.

Theorem 3 depends on the following refinement of Lemma 2.

Lemma 3. Let $\alpha$ be in Bad. Let a be a real number with $0<a<1$. There exists a positive constant $C(\alpha)$ such that, for every integer $q \geq 2$, we have

$$
\sum_{x=q}^{q^{3}} \frac{1}{\|\alpha x\| x(\log 1 /\|x \alpha\|)^{a} \cdot(\log x)^{2-a}} \leq C(\alpha) .
$$

Proof. Let $\left(p_{j} / q_{j}\right)_{j \geq 0}$ denote the sequence of convergents to $\alpha$. Let $m$ (resp. $n$ ) be the largest (resp. the smallest) integer $j$ such that $q_{j} \leq q$ (resp. $q_{j} \geq q^{3}$ ). As the sequence $\left(q_{j}\right)_{j \geq 0}$ grows exponentially fast, we have

$$
\log q \ll n \ll m \ll \log q
$$

where, as throughout this proof, the numerical constants implied by $\ll$ depend only on $\alpha$.

Let $j$ be an integer satisfying $m \leq j<n$ and consider

$$
S_{j}:=\sum_{x=q_{j}}^{q_{j+1}} \frac{1}{\|\alpha x\| x(\log 1 /\|x \alpha\|)^{a}} .
$$

A classical result asserts that the points $\{\alpha x\}, x=1, \ldots, q_{j+1}$, are very well distributed in $(0,1)$. Consequently,

$$
\begin{aligned}
S_{j} \ll \frac{1}{q_{j}} \sum_{x=1}^{q_{j+1}} \frac{1}{\|\alpha x\|(\log 1 /\|x \alpha\|)^{a}} & \ll \frac{1}{q_{j}} \sum_{x=1}^{q_{j+1} / 2} \frac{\left(q_{j+1} / x\right)}{\left(\log \left(q_{j+1} / x\right)\right)^{a}} \\
& \ll \frac{q_{j+1}}{q_{j}} \int_{2}^{q_{j+1}} \frac{d u}{u(\log u)^{1-a}} \ll\left(\log q_{j}\right)^{1-a},
\end{aligned}
$$

since $q_{j+1} / q_{j}$ is bounded from above by an absolute constant depending only on $\alpha$. Now,

$$
\sum_{j=m}^{n} S_{j} \ll(\log q)^{2-a},
$$

which proves the lemma.

The key tool for the proof of Theorem 5 is Lemma 4 below. 
Lemma 4. Let $p$ be a prime number. Let $a$ be a real number with $0 \leq a<1$. There exists a positive constant $C(a, p)$ such that, for every integer $q \geq 2$, we have

$$
\sum_{x=q}^{q^{3}} \frac{1}{x \cdot|x|_{p}\left(\log \left(2 /|x|_{p}\right)\right)^{a} \cdot(\log x)^{2-a}} \leq C(a, p) .
$$

Proof. Observe that

$$
\sum_{x=q}^{q^{3}} \frac{1}{x \cdot|x|_{p} \cdot\left(\log \left(2 /|x|_{p}\right)\right)^{a}} \ll \sum_{j=0}^{3 \log q} \sum_{x=\left\lceil q / p^{j}\right\rceil}^{\left\lfloor q^{3} / p^{j}\right\rfloor} \frac{1}{x(j+1)^{a}},
$$

where the second summation is taken over the integers $x$ that are not divisible by $p$. Consequently,

$$
\sum_{x=q}^{q^{3}} \frac{1}{x \cdot|x|_{p} \cdot\left(\log \left(2 /|x|_{p}\right)\right)^{a}} \ll \sum_{j=0}^{3 \log q} \frac{\log q}{(j+1)^{a}} \ll(\log q)^{2-a}
$$

and the lemma is proved.

\section{Proof of Theorem 1}

Let $\alpha$ be in $\boldsymbol{B a d}$ and $\delta$ be a positive real number satisfying

$$
q \cdot\|q \alpha\| \geq \delta, \quad \text { for every } q \geq 1 \text {. }
$$

Let $\varepsilon$ be such that

$$
0<\varepsilon<\left(2^{10} C(\alpha)\right)^{-1},
$$

where $C(\alpha)$ is given by Lemma 2 .

We follow a method introduced by Peres and Schlag [23]. First, we construct 'dan-

gerous' sets of real numbers. These sets depend on $\alpha$, but, to simplify the notation, we choose not to indicate this dependence. For integers $x$ and $y$ with $x \geq 2$ and $0 \leq y \leq x$, define

$$
E(x, y)=\left[\frac{y}{x}-\frac{\varepsilon}{\|\alpha x\| x^{2} \log _{2}^{2} x}, \frac{y}{x}+\frac{\varepsilon}{\|\alpha x\| x^{2} \log _{2}^{2} x}\right]
$$

and

$$
E(x)=\bigcup_{y=0}^{x}(E(x, y) \cap[0,1]) .
$$

Set also

$$
l_{0}=0, \quad l_{x}=\left\lfloor\log _{2}\left(\|\alpha x\| x^{2} \log _{2}^{2} x /(2 \varepsilon)\right)\right\rfloor, \quad \text { for } x \in \mathbf{Z}_{\geq 1} .
$$


Each interval from the union $E(x)$ defined in (4.4) can be covered by an open dyadic interval of the form

$$
\left(\frac{b}{2^{l_{x}}}, \frac{b+z}{2^{l_{x}}}\right), \quad z=1,2, \quad b \in \mathbf{Z}_{\geq 0} .
$$

Let $A(x)$ be the smallest union of all such dyadic intervals which covers the whole set $E(x)$ and put

$$
A^{c}(x)=[0,1] \backslash A(x) .
$$

Observe that $A^{c}(x)$ is a union of closed intervals of the form

$$
\left[\frac{a}{2^{l_{x}}}, \frac{a+1}{2^{l_{x}}}\right], a \in \mathbf{Z}_{\geq 0} .
$$

Let $q_{0}$ be an integer such that

$$
q_{0} \geq(100 \varepsilon)^{3} \quad \text { and } \quad\left\|q_{0} \alpha\right\| \geq 1 / 4 .
$$

For $q \geq q_{0}$, define

$$
B_{q}=\bigcap_{x=q_{0}}^{q} A^{c}(x) .
$$

The sets $B_{q}, q \geq q_{0}$, are closed and nested. Our aim is to show inductively that they are non-empty. Set $L_{0}=l_{0}$ and

$$
q_{k}:=q_{0}^{3^{k}}, \quad L_{k}=\left\lfloor\log _{2}\left(q_{k}^{2} \log _{2}^{2} q_{k} /(4 \varepsilon)\right)\right\rfloor, \quad k \geq 1 .
$$

Observe that $l_{x} \leq L_{k}$ when $x \leq q_{k}$.

For every integer $k \geq 0$ we construct inductively subsets $C_{q_{k}}$ and $D_{q_{k}}$ of $B_{q_{k}}$ with the following property $\left(P_{k}\right)$ :

The set $C_{q_{k}}$ is the union of $2^{-5 k-3+L_{k}}$ intervals of length $2^{-L_{k}}$, separated by at least $2^{-L_{k}}$, and such that at least $2^{-5 k-5+L_{k}}$ among them include at least $2^{L_{k+1}-L_{k}-3}$ intervals composing $B_{q_{k+1}}$, which are also separated by at least $2^{-L_{k+1}}$. Let denote by $C_{q_{k+1}}$ (resp. by $D_{q_{k}}$ ) the union of $2^{-5(k+1)-3+L_{k+1}}$ of these intervals (resp. of the corresponding $2^{-5 k-5+L_{k}}$ intervals from $C_{q_{k}}$ ). In particular, we have mes $C_{q_{k}}=4 \operatorname{mes} D_{q_{k}}=2^{5} \operatorname{mes} C_{q_{k+1}}$.

We deduce from (4.2), (4.3) and Lemma 2 that

$$
\operatorname{mes}\left(B_{q_{1}}\right) \geq 1-\sum_{x=q_{0}}^{q_{1}} \operatorname{mes} A(x) \geq 31 / 32 .
$$

Consequently, $B_{q_{1}}$ is the union of at least $2^{L_{1}-1}$ intervals of length $2^{-L_{1}}$. By (4.6), the set $B_{q_{0}}$ is the union of at least $2^{L_{0}-1}$ intervals of length $2^{-L_{0}}$. This allows us to define the sets $C_{q_{0}}, D_{q_{0}}$ and $C_{q_{1}}$. This proves $\left(P_{0}\right)$. 
Let $k$ be a non-negative integer such that $\left(P_{k}\right)$ holds, and consider the set $B_{q_{k+2}}^{\prime}:=$ $C_{q_{k+1}} \cap B_{q_{k+2}}$. Observe that

$$
B_{q_{k+2}}^{\prime}=C_{q_{k+1}} \backslash\left(\bigcup_{x=q_{k+1}+1}^{q_{k+2}} A(x)\right)
$$

hence

$$
\operatorname{mes} B_{q_{k+2}}^{\prime} \geq \operatorname{mes} C_{q_{k+1}}-\sum_{x=q_{k+1}+1}^{q_{k+2}} \operatorname{mes}\left(C_{q_{k+1}} \cap A(x)\right) .
$$

By construction, the set $C_{q_{k}}$ can be written as a union, say

$$
C_{q_{k}}=\bigcup_{\nu=1}^{T_{q_{k}}} J_{\nu}
$$

of $T_{q_{k}}$ dyadic intervals $J_{\nu}$ of the form

$$
\left[\frac{a}{2^{L_{k}}}, \frac{a+1}{2^{L_{k}}}\right], \quad a \in \mathbf{Z}_{\geq 0},
$$

where $L_{k}$ is given by (4.7). Let $x \geq q_{k}^{3}$ be an integer. Since, by (4.6),

$$
2^{L_{k}} \leq \frac{q_{k}^{2} \log _{2}^{2} q_{k}}{4 \varepsilon} \leq \frac{q_{k}^{3}}{2} \leq \frac{x}{2},
$$

each interval $J_{\nu}$ contains at least the rationals $y / x,(y+1) / x$ for some integer $y$, and we infer from (4.3) that

$$
\operatorname{mes}\left(J_{\nu} \cap A(x)\right) \leq \frac{2^{4} \varepsilon}{\|\alpha x\| x \log _{2}^{2} x} \times \operatorname{mes} J_{\nu}
$$

Summing (4.9) from $\nu=1$ to $\nu=T_{q_{k}}$, we get

$$
\operatorname{mes}\left(C_{q_{k}} \cap A(x)\right) \leq \frac{2^{4} \varepsilon}{\|\alpha x\| x \log _{2}^{2} x} \times \operatorname{mes} C_{q_{k}} .
$$

It then follows from (4.10) that

$$
\begin{aligned}
\operatorname{mes}\left(C_{q_{k+1}} \cap A(x)\right) & \leq \operatorname{mes}\left(C_{q_{k}} \cap A(x)\right) \\
& \leq \frac{2^{4} \varepsilon}{\|\alpha x\| x \log _{2}^{2} x} \times \operatorname{mes} C_{q_{k}} \leq \frac{2^{9} \varepsilon}{\|\alpha x\| x \log _{2}^{2} x} \times \operatorname{mes} C_{q_{k+1}} .
\end{aligned}
$$

Combined with (4.8) and Lemma 2, this gives

$$
\operatorname{mes} B_{q_{k+2}}^{\prime} \geq\left(\operatorname{mes} C_{q_{k+1}}\right)\left(1-\sum_{x=q_{k+1}+1}^{q_{k+2}} \frac{2^{9} \varepsilon}{\|\alpha x\| x \log _{2}^{2} x}\right) \geq \frac{\operatorname{mes} C_{q_{k+1}}}{2} .
$$


Thus, at least one quarter of the intervals composing $C_{q_{k+1}}$ contains at least $2^{L_{k+2}-L_{k+1}-2}$ intervals composing $B_{q_{k+2}}^{\prime}$, thus at least $2^{L_{k+2}-L_{k+1}-3}$ intervals composing $B_{q_{k+2}}^{\prime}$, if we impose that these intervals are mutually distant by at least $2^{-L_{k+2}}$. This allows us to define the sets $C_{q_{k+2}}$ and $D_{q_{k+1}}$ with the required properties. This proves $\left(P_{k+1}\right)$.

It then follows that the set

$$
\mathcal{K}:=\bigcap_{k \geq 0} D_{q_{k}}
$$

is non-empty. By construction, every point $\beta$ in this set avoids all the intervals $E(x, y)$ with $x \geq q_{0}$, thus, the pair $(\alpha, \beta)$ satisfies $(2.1)$.

To establish that the set $\mathcal{K}$ has full Hausdorff dimension, we apply Lemma 1 with

$$
m_{k}=2^{L_{k+1}-L_{k}-5} \quad \text { and } \quad \varepsilon_{k}:=2^{-L_{k+1}} .
$$

Note that

$$
\frac{\log \left(m_{1} \ldots m_{k-1}\right)}{-\log \left(m_{k} \varepsilon_{k}\right)} \geq \frac{\log \left(32^{-k} 2^{L_{k}}\right)}{-\log \left(2^{-L_{k}+5}\right)}
$$

We infer from (4.1), (4.5) and (4.7) that

$$
2^{L_{k}} \geq \delta q_{0}^{3^{k}}
$$

Consequently,

$$
\lim _{k \rightarrow+\infty} \frac{\log \left(m_{1} \ldots m_{k-1}\right)}{-\log \left(m_{k} \varepsilon_{k}\right)}=1
$$

and it follows from Lemma 1 that the set $\mathcal{K}$ has full Hausdorff dimension. This completes the proof of our theorem.

\section{Proofs of Theorems 3, 4, and 5}

The proofs of Theorems 3 and 5 follow exactly the same steps as that of Theorem 1 . Instead of the intervals

$$
E(x, y)=\left[\frac{y}{x}-\frac{\varepsilon}{\|\alpha x\| x^{2} \log _{2}^{2} x}, \frac{y}{x}+\frac{\varepsilon}{\|\alpha x\| x^{2} \log _{2}^{2} x}\right],
$$

we use respectively the intervals

$$
\left[\frac{y}{x}-\frac{\varepsilon}{\|\alpha x\| x^{2}\left(\log _{2} x\right)^{2-a}(\log 1 /\|\alpha x\|)^{a}}, \frac{y}{x}+\frac{\varepsilon}{\|\alpha x\| x^{2}\left(\log _{2} x\right)^{2-a}(\log 1 /\|\alpha x\|)^{a}}\right]
$$

and

$$
\left[\frac{y}{x}-\frac{\varepsilon}{|x|_{p} x^{2}\left(\log _{2} x\right)^{2-a}\left(\log 2 /|x|_{p}\right)^{a}}, \frac{y}{x}+\frac{\varepsilon}{|x|_{p} x^{2}\left(\log _{2} x\right)^{2-a}\left(\log 2 /|x|_{p}\right)^{a}}\right] .
$$


Furthermore, we apply Lemmas 3 and 4 in place of Lemma 2.

For the proof of Theorem 4, we work directly in the plane. The idea is the following. For a triple $(x, y, z)$ of integers and a positive $\varepsilon$, the inequality $|x X+y Y+z| \leq \varepsilon$ defines a strip composed of points $(X, Y)$ close to the line $x X+y Y+z=0$. Since we are working in the unit square, to a given pair $(x, y)$ of integers corresponds a unique $z$, and the length of the intersection of the line with the unit square is at most equal to $\sqrt{2}$. Setting

$$
\varepsilon_{x, y}=\frac{\varepsilon}{|x y| \log ^{2}|x y|},
$$

for a given (very small) positive $\varepsilon$, the strips $|x X+y Y+z| \leq \varepsilon_{x, y}$ play the same role as the intervals (4.3) in the proof of Theorem 1.

Since, for every large integer $q$, we have

$$
\begin{aligned}
\sum_{q \leq x y \leq q^{3}} \varepsilon_{x, y} & \ll \sum_{x=1}^{q^{3}} \sum_{y=\lfloor q / x\rfloor}^{\left\lfloor q^{3} x\right\rfloor} \frac{\varepsilon}{|x y| \log ^{2} q} \\
& \ll \sum_{x=1}^{q^{3}} \frac{\varepsilon}{\log q} \ll \varepsilon,
\end{aligned}
$$

the Peres-Schlag method can be applied as in the proof of Theorem 1. We omit the details.

\section{Further results}

We gather in the present section several results that can be obtained with the same method as in the proof of Theorem 1 .

* A result on lacunary sequences.

Theorem 6. Let $M$ be a positive real number and $\left(t_{j}\right)_{j \geq 1}$ be a sequence such that $t_{j+1} / t_{j}>1+1 / M$ for $j \geq 1$. Let $c$ be a real number with $0<c<1 / 10$. Let $\varepsilon$ be a positive real number. Then, the Hausdorff dimension of the set

$$
\left\{\xi \in[0,1]: \forall n \geq 1,\left\|\xi t_{n}\right\| \geq c /(M \log M)\right\} .
$$

is at least $1-\varepsilon$ if $M$ is sufficiently large.

Theorem 6 complements the results from $[23,17]$.

* The use of the mass distribution principle enables us to improve Theorem 1 of [19].

Theorem 7. Let $C_{1}, C_{2}$ and $\gamma$ be positive real numbers. Let $\left(t_{n}\right)_{n \geq 1}$ be a sequence of real numbers such that

$$
C_{1} n^{\gamma} \leq t_{n} \leq C_{2} n^{\gamma}, \quad \text { for } n \geq 1 \text {. }
$$


Then, there exist a positive $C$ and an integer $n_{0}$ such that the set

$$
\bigcap_{n \geq n_{0}}\left\{\xi \in \mathbf{R}:\left\|\xi t_{n}\right\|>\frac{C}{n \log n}\right\}
$$

has full Hausdorff dimension.

It is established in [19] that the Hausdorff dimension of the set $(6.1)$ is at least $\gamma /(\gamma+1)$. As an immediate application, we get that the set of real numbers $\xi$ for which

$$
\liminf _{n \rightarrow+\infty} n(\log n)\left\|\xi n^{2}\right\|>0
$$

has full Hausdorff dimension.

* We have stated homogeneous statements, but the method as well allows us to deal with inhomogeneous approximation.

* By means of dyadic arguments as it was done in the preprint [21], it is possible to generalize Lemmas 2 and 3 and, eventually, to establish the following statement.

Theorem 8. Consider real paremeters $A>1,0<\varepsilon<1$ and $\delta>0$. Let $\alpha$ be a badly approximable real number, such that

$$
\inf _{q \geq 1} q\|q \alpha\| \geq \delta>0
$$

Consider real-valued functions $\psi_{j}, j=0,1,2$, defined over the non-negative real numbers, satisfying tho following conditions:

$$
\psi_{0}(x)>0 \text { for } x \text { large enough and } \Psi_{0} \text { is increasing; }
$$

$$
\psi_{j}(0)=0, \quad j=1,2
$$

(iii) $\quad \psi_{j}$ increases in some interval of the type $[0, \xi], \xi>0$ and $\max _{0 \leq x \leq \xi} \psi_{j}(x) \leq 1$;

$$
\max _{x \in \mathbf{N}} \psi_{0}(x) \psi_{2}\left(x^{1-A}\right) \leq \varepsilon
$$

Define $\psi_{2}^{-1}(x)$ to be the inverse function to $\psi_{2}(x)$ so $\psi_{2}^{-1}\left(\psi_{2}(x)\right)=x$. Suppose that

$$
\sup _{X \in \mathbf{N}} \sum_{X \leq \nu<A X} \sum_{1 \leq \mu \leq \nu+2-\log _{2} \delta} 2^{\nu-\mu} \times \psi_{2}^{-1}\left(\frac{\varepsilon}{\psi_{0}\left(2^{\nu}\right) \psi_{1}\left(2^{-\mu}\right)}\right) \leq \frac{1}{2^{6}} .
$$


Take an arbitrary sequence of reals $\left(\eta_{q}\right)_{q \geq 1}$. Then there exists a real number $\beta$ such that

$$
\liminf _{q \rightarrow+\infty} \psi_{0}(q) \psi_{1}(\|q \alpha\|) \psi_{2}\left(\left\|q \beta+\eta_{q}\right\|\right)>\varepsilon
$$

The proof of Theorem 8 follows directly the arguments from [21].

For a real number $a$ with $0 \leq a<1$, if we put

$$
\psi_{0}(x)=x \log ^{2-a} x, \psi_{1}(x)=x(\log 1 / x)^{a}, \psi_{2}(x)=x, \quad \eta_{q}=0, q \geq 1,
$$

then Theorem 8 implies that there exists a real number $\beta$ such that

$$
\liminf _{q \rightarrow+\infty} q \cdot(\log q)^{2-a} \cdot(\log 1 /\|q \alpha\|)^{a} \cdot\|q \alpha\| \cdot\|q \beta\|>0
$$

a result which corresponds to Theorem 3 (and to Theorem 1 if $a=0$ ), with the exception of the assertion on the Hausdorff dimension. Unfortunately, we cannot put here $a=1$.

\section{References}

[1] B. Adamczewski and Y. Bugeaud, On the Littlewood conjecture in simultaneous Diophantine approximation, J. London Math. Soc. 73 (2006), 355-366.

[2] V. V. Beresnevich and S. L. Velani, A note on simultaneous Diophantine approximation on planar curves, Math. Ann. 337 (2007), 769-796.

[3] V. Bernik, D. Kleinbock, and G. A. Margulis, Khintchine-type theorems on manifolds: the convergence case for standard and multiplicative versions, Internat. Math. Res. Notices (2001), 453-486.

[4] Y. Bugeaud, Approximation by algebraic numbers, Cambridge Tracts in Mathematics 160, Cambridge, 2004.

[5] Y. Bugeaud, M. Drmota, and B. de Mathan, On a mixed Littlewood conjecture in Diophantine approximation, Acta Arith. 128 (2007), 107-124.

[6] Y. Bugeaud, A. Haynes, and S. Velani, Metric considerations concerning the mixed Littlewood conjecture. In preparation.

[7] J. W. S. Cassels and H. P. F. Swinnerton-Dyer, On the product of three homogeneous linear forms and indefinite ternary quadratic forms, Philos. Trans. Roy. Soc. London, Ser. A, 248 (1955), 73-96.

[8] M. Einsiedler, A. Katok, and E. Lindenstrauss, Invariant measures and the set of exceptions to the Littlewood conjecture, Ann. of Math. 164 (2006), 513-560.

[9] M. Einsiedler and D. Kleinbock, Measure rigidity and p-adic Littlewood-type problems, Compositio Math. 143 (2007), 689-702. 
[10] K. Falconer, Fractal geometry. Mathematical foundations and applications. John Wiley \& Sons, Ltd., Chichester, 1990.

[11] P. Gallagher, Metric simultaneous Diophantine aproximations, J. London Math. Soc. 37 (1962), $387-390$.

[12] V. Jarník, Zur metrischen Theorie der diophantischen Approximationen, Práce Mat.Fiz. 36 (1928/29), 91-106.

[13] L. Kuipers and H. Niederreiter, Uniform distribution of sequences. Pure and Applied Mathematics. Wiley-Interscience [John Wiley \& Sons], New York-London-Sydney, 1974 .

[14] J. E. Littlewood, Some problems in real and complex analysis. D. C. Heath and Co. Raytheon Education Co., Lexington, Mass., 1968.

[15] B. de Mathan, Conjecture de Littlewood et récurrences linéaires, J. Théor. Nombres Bordeaux 13 (2003), 249-266.

[16] B. de Mathan et O. Teulié, Problèmes diophantiens simultanés, Monatsh. Math. 143 (2004), 229-245.

[17] N. G. Moshchevitin, A version of the proof for Peres-Schlag's theorem on lacunary sequences. Available at arXiv: 0708.2087v2 [math.NT] 15Aug2007.

[18] N. G. Moshchevitin, Density modulo 1 of sublacunary sequences: application of Peres-Schlag's arguments. Preprint, available at arXiv: 0709.3419v2 [math.NT] 20 Oct2007

[19] N. G. Moshchevitin, On small fractional parts of polynomials, J. Number Theory 129 (2009), 349-357.

[20] N. G. Moshchevitin, Towards BAD conjecture. Available at arXiv: 0712.2423v2 12 Apr2008.

[21] N. G. Moshchevitin, Badly approximable numbers related to the Littlewood conjecture. Preprint, available at arXiv: 0810.0777.

[22] L. G. Peck, Simultaneous rational approximations to algebraic numbers, Bull. Amer. Math. Soc. 67 (1961), 197-201.

[23] Yu. Peres and W. Schlag, Two Erdös problems on lacunary sequences: chromatic numbers and Diophantine approximations. Available at: arXiv: 0706.0223v1.

[24] A. D. Pollington and S. Velani, On a problem in simultaneous Diophantine approximation: Littlewood's conjecture, Acta Math. 185 (2000), 287-306. 
Yann Bugeaud

Université Louis Pasteur

Mathématiques

7, rue René Descartes

67084 STRASBOURG Cedex (France)

bugeaud@math.u-strasbg.fr
Nikolay Moshchevitin

Moscow State University

Number Theory

Leninskie Gory 1

MOSCOW (Russian federation)

moshchevitin@rambler.ru 